\title{
Enhancing students' mathematical reasoning in the classroom: teacher actions facilitating generalization and justification
}

\author{
Joana Mata-Pereira \\ jmatapereira@ie.ulisboa.pt \\ Instituto de Educação, Universidade de Lisboa, Portugal
}

\author{
João-Pedro da Ponte \\ jpponte@ie.ulisboa.pt \\ Instituto de Educação, Universidade de Lisboa, Portugal
}

\begin{abstract}
A proof is a connected sequence of assertions that includes a set of accepted statements, forms of reasoning and modes of representing arguments. Assuming reasoning to be central to proving and aiming to develop knowledge about how teacher actions may promote students' mathematical reasoning, we conduct design research where whole-class mathematical discussions triggered by exploratory tasks play a key role. We take mathematical reasoning as making justified inferences and we consider generalizing and justifying central reasoning processes. Regarding teacher actions, we consider inviting, informing/suggesting, supporting/guiding and challenging actions can be identified in whole-class discussions. This paper presents design principles for an intervention geared to tackle such reasoning processes and focuses on a whole-class discussion on a grade 7 lesson about linear equations and functions. Data analysis concerns teacher actions in relation to design principles and to the sought mathematical reasoning processes. The conclusions highlight teacher actions that lead students to generalize and justify. Generalizations may arise from a central challenging action or from several guiding actions. Regarding justifications, a main challenging action seems to be essential, while followup guiding actions may promote a further development of this reasoning process. Thus, this paper provides a set of design principles and a characterization of teacher actions which enhance students' mathematical reasoning processes such as generalization and justification.
\end{abstract}

Keywords Mathematical reasoning . Teacher actions. Generalization .Justification . Design research

\section{Introduction}

Engaging students in proving is a major challenge in school mathematics. Proving, the activity of making a proof, consists of mathematically arguing by sequencing assertions for or against a mathematical claim (Stylianides 2007). As indicated by Stylianides (2007), in order to be considered a proof, this connected sequence of assertions must include a set of accepted statements (e.g., definitions, theorems), forms of reasoning and modes of argument representation. In this paper, we focus on mathematical reasoning as a central element of students' proving activity.

Mathematical reasoning, besides providing students with conditions to engage in proving, allows them to go beyond the routine use of procedures towards learning concepts, properties, and procedures as logical, interrelated, and coherent aspects of mathematics. As such, developing students' mathematical reasoning in everyday classrooms is a core aspect of teaching and learning mathematics (Ball and Bass 2003; Boaler 2010). However, to promote students' mathematical reasoning, teachers need to provide challenging learning environments rather than just lessons where they solve exercises using well-known procedures. We take whole class mathematical discussions triggered by exploratory tasks (Ruthven 1989) 
as privileged moments to create opportunities to develop students' mathematical reasoning, especially the central reasoning processes of generalization and justification (Lannin, Ellis and Elliot 2011).

In this paper, we aim to develop knowledge about how teacher actions may enhance students' mathematical reasoning, an issue that we address by conducting a design-based research (Cobb et al. 2003). Thus, we design principles to support teachers in enhancing students' mathematical reasoning and conduct an intervention based on those principles. We illustrate and analyze this intervention through the lenses of characteristics of tasks, teacher actions, and students' generalization and justification processes. Therefore, this research-based intervention study (informed by theory and empirical evidence) aims both to contribute with a set of principles regarding tasks and teachers' actions designed to enhance students' mathematical reasoning and to illustrate how those principles might be enacted in the classroom and enhance students' mathematical reasoning.

\section{Developing students' mathematical reasoning}

\subsection{Mathematical reasoning and reasoning processes}

"Reasoning" is a very common term in mathematics education, but often with an imprecise meaning, close or synonymous of "thinking". We hereby consider mathematical reasoning as making justified inferences (Brousseau and Gibel 2005; Pólya 1954; Rivera and Becker 2009), using deductive, inductive, and abductive processes. Thus, mathematical reasoning processes include formulating questions and solving strategies, formulating and testing generalizations and other conjectures, and justifying them. We focus on generalizing and justifying as key mathematical reasoning processes.

Conjecturing, is to state something that is intended to be true, but that is still not known as such (Lannin et al. 2011). An important way of conjecturing, mathematical generalizing, is a process that leads to a statement referring to a common property of a group of objects (Dörfler 1991). Generalizing also comprises stating that a property or procedure, known for a certain set of objects or conditions, holds for a larger set of objects or conditions (Carraher, Martinez and Schliemann 2008). Being the foundation of mathematical concepts and ideas, this is a central reasoning process. It is essential to engage students in situations that promote generalizations since much mathematical learning may develop from these activities (Kieran, 2007). However, we must note that generalizations in the classroom may be unstated or incorrect (Becker and Rivera 2005; Reid 2002) and thus setting the need for elaboration and justification.

Justifying a claim or a result involves presenting reasons convincing to oneself and others (Sowder and Harel 1998). In order to do so, justifications need to rely on accepted concepts, properties, procedures, and mathematical ideas. Thus, it is essential that students understand the need for justification from very early in their schooling. Therefore, as Lannin et al. (2011) highlight, students should engage on justifying by relying on previously understood mathematical ideas or refuting statements by providing counter-examples. As these authors refer, it is also important that students develop knowledge about what validates an argument, rejecting arguments that depend on authority, perception, common sense, or just particular cases.

Students must be able to justify why a generalization is valid or not. As they advance through their schooling, the perception about what validates and invalidates a justification should lead to increasingly more formal justifications. These formal justifications, as they present mathematical reasons 
to support a claim, are often equivalent to proofs or a significant part of proofs. Thus, to prompt justifications is a fundamental move towards proving.

\subsection{Tasks and teacher actions}

Mathematical reasoning requires that students get involved in a variety of thinking and sense making processes. We may obtain traces of students' mathematical reasoning from their classroom work and from classroom interactions (Brodie 2010). Therefore, two basic elements of teacher professional practice are at stake: the tasks proposed to the students and the communication processes enhanced by teacher actions (Ponte and Quaresma 2016). Appropriate tasks are essential to support students' mathematics learning in the classroom (Brodie 2010; Christiansen and Walter 1986), in particular tasks that aim at developing mathematical reasoning. Therefore, it is necessary to know which tasks lead students to engage in mathematical reasoning, and in what ways they may be used in the classroom (Brodie 2010). Previous research identified problems and exploration and investigation tasks as means to develop students' mathematical reasoning (e.g., Francisco and Maher 2011). Also in this perspective, Ponte and Quaresma (2016) indicate that challenging tasks are a fertile ground to promote mathematical reasoning. However, proposing too many challenging tasks in a limited time may easily demotivate and disinterest many students (Brodie 2010). It is also important to propose tasks with a lower level of challenge aiming students to easily engage in the classroom activity. Also, tasks that lead to multiple representations or solving processes or that promote disagreements, may help to develop students' mathematical reasoning (Brodie 2010).

Notwithstanding their importance suitable tasks are not enough to ensure that students develop mathematical reasoning (Ball and Bass 2003). Within the complexity of professional practice, a key aspect to consider is teacher actions (sometimes also referred to as "teacher moves"). These teacher actions originate from the teacher's motives regarding the nature of the sought classroom activity (Christiansen and Walter 1986) and are central to achieve them. For instance, if the teacher gives students too many indications and suggestions, the task is simplified and solving it becomes a simple exercise, not requiring much reasoning. Also, if the teacher stays aside while students work, he/she may fail to provide the necessary support for students to carry out their reasoning (Brodie 2010). In classrooms marked by exploratory teaching (Ruthven 1989), whole class discussions have a strong potential to foster students' learning (Ponte 2005). As these discussions yield students opportunities to share, discuss, and evaluate their ideas they are also propitious to develop mathematical reasoning. In these discussions, teacher actions should value arguments that address the "why" of results and not just the results themselves (Sowder and Harel 1998). As Brodie (2010) indicates, the teacher should broaden and explore students' proper contributions and, if acceptable, value students' incorrect or partial contributions seeking that connections to the solution and to the meaning of concepts and mathematical ideas are made. Wood (1999) also points out that the teacher may explore disagreements between students, as such situations may develop their ability to justify. In order to do so, the teacher should create opportunities for students to consider others' ideas and critically evaluate their validity (Ball and Bass 2003). As such, the role of the teacher is to articulate students' contributions and advance the discussion leading them to the sought mathematical ideas (Stein, Engle, Smith and Hughes 2008). 
Conducting those discussions is particularly complex as it often leads to unexpected student contributions. The teacher has to support both the content of the discussion and its management. Namely he/she has to be able to articulate multiple ideas that emerge from the students, to encourage students to elaborate their thinking, to lead them to evaluate and compare their ideas and often also to guarantee that important mathematical ideas are the focus of the classroom activity (Sherin 2002). A single question (either specific or general) is often not enough to uncover enough detail of students' reasoning processes, thus requiring a sequence of actions on following up students' ideas (Franke et al., 2009). This sequence of actions may encompass generating discussion, by requesting students' participation; orienting and focusing, by requesting for clarification or elaboration, providing a piece of information, a hint or a direction; and challenging or probing students (Kosko, Rougee and Herbst 2014; Krussel, Edwards and Springer 2004).

Within this scope of teacher actions, Ponte and Quaresma (2016) suggest a model (Figure 1) that distinguishes between actions directly related to mathematical topics and processes and actions related to management of learning. Regarding actions related to mathematical processes, inviting actions aim to trigger a whole class discussion or a discussion segment, where the teacher encourages students to participate or share their responses. Then, the teacher relies mostly on informing/suggesting actions to provide information to students or to validate their statements; on supporting/guiding actions to lead students to present information; and on challenging actions to encourage students to go further than their previous knowledge. In those three sets of actions, the authors refer several mathematical processes that are involved, not necessarily disjoint, such as representing, interpreting, reasoning, and evaluating. Regarding these mathematical processes, in this paper we focus only on reasoning using this model as a lens towards building elements for enhancing mathematical reasoning.

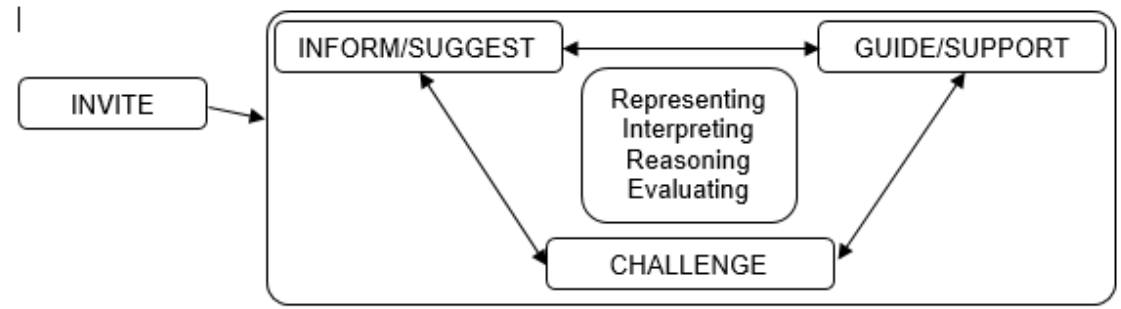

Fig. 1 Model to analyze teacher actions (adapted from Ponte and Quaresma 2016).

\section{The design experiment}

This research is conducted through a design experiment (Cobb et al. 2003) entailed to provide teachers the means to develop students' mathematical reasoning and researchers the tools to systematically analyze those means. In order to do so, design principles, i.e., heuristics that structure the intervention, focusing on tasks and teacher actions are drawn from the research literature and from a previous cycle of experimentation.

\subsection{Design principles}

Attending to the issues presented concerning tasks, to the reasoning processes that we highlight (generalization and justification), and to the results of a previous cycle of this design-based research, we formulate four general principles related to tasks. These principles indicate that tasks should include: (i) a 
diversity of questions of different levels of challenge, emphasizing problems and exploratory questions but also exercises; (ii) questions that prompt generalizations; (iii) questions that ask for justification of answers and of solving processes; and (iv) questions that allow a variety of solving processes.

Based both on the literature reviewed and on the previous experimentation cycle, we also consider some principles regarding teacher actions that aim to structure the intervention: (a) monitor students while they solve the task, aiming not to reduce its level of challenge; (b) ask students to explain "why" and to present alternative justifications; (c) highlight or ask students to identify valid and invalid justifications, emphasizing what may validate them; (d) encourage the sharing of ideas; (e) accept and value incorrect or partial contributions by deconstructing, supplementing, or clarifying them; (f) support or inform students in order to highlight reasoning processes such as generalizing and justifying, and (g) challenge students to go beyond the task by formulating new questions, generalizing or justifying.

Accepting these design principles regarding tasks and teacher actions is one thing, and implementing them in the classroom in ways that promote students' mathematical reasoning is another one, involving a significant challenge to the teacher. Thus, instead of attempting to impose from the outset certain tasks or teacher actions, we discuss all these elements with the teacher regarding how to create conditions to promote students' learning and the development of mathematical reasoning. This approach informs the intervention and allows to relate assumptions in the literature on the development of students' mathematical reasoning with new ideas regarding tasks and teacher actions. As Boaler (2010) suggests, these connections between the literature and teaching experience may bring powerful results.

\subsection{Participants, context, and data collection}

The intervention took place in a grade 7 class of a public school in Portugal, throughout four 90-minute lessons and five 45-minute lessons about linear equations. An algebraic topic was chosen since algebra is a powerful field to support mathematical reasoning. This intervention concerns the second cycle of the design-based research, after a first cycle that took place in lessons about sequences also aiming to develop students' mathematical reasoning. Given the nature of this design experiment, the participating teacher was selected by her exploratory teaching approach, her availability to consider changes in her practice, and her confidence to collaborate based on her teaching experience. Together with the teacher, the first author planned a unit about linear equations based on the design principles. Earlier in the year, the students had lessons about linear functions, with no participation from the researchers. Most tasks were proposed by the first author and then discussed and adjusted with the teacher in order to fit the class characteristics. Aiming to appropriately address the design principles during the intervention, the first author and the teacher had working sessions after each lesson. In these working sessions, a detailed plan of each lesson was also prepared with the teacher's contributions. Each lesson plan addressed learning goals, general strategy, lesson structure, resources to use, tasks, and learning activities, as well as expected students' responses and difficulties, expected teacher answers to those responses and difficulties, and aims and evaluation of each moment of the lesson. In order to inform this research, interviews were conducted prior to and after those nine lessons to both the teacher and selected students. All lessons were directly observed and all the interviews, lessons and working sessions were video and audio recorded and notes made in a researcher's logbook. 
All participant students in this study are volunteer and had the right to withdraw from it at any moment. An informed consent was requested to all participants and, when appropriate, to their legal guardians. In order to respect their anonymity, all their names are fictitious.

\subsection{Data analysis}

Aiming to identify ways to promote students' mathematical reasoning, data analysis is centered on teacher actions during whole class discussions (presented in Figure 1) and their relations with the design principles for tasks and for teacher actions. Data analysis is made with support from Nvivo software where video and audio records were categorized regarding teacher actions, mathematical reasoning processes, and design principles. The episodes presented in this paper are taken from lesson eight of the nine lessons that constitute the unit and aimed to lead students to relate equations and functions. These episodes are chosen from this lesson since they illustrate a variety of situations in which we identify reasoning processes and enable an analysis of the relations between design principles and teacher actions.

\section{When functions precede equations: a grade 7 case}

Taking into account the design principles, the task (Figure 2) includes as a first question an exercise, as students already had the tools to reach the solution, and two other exploratory questions that aim to be more challenging and lead students to advance their knowledge (principle (i)). Question 1 provides an opportunity for students to easily engage in the task, and also aims the discussion and review of some concepts and ideas regarding affine functions, namely, the possible meaning of $x, m(x)$ and $j(x)$, the needed information in a complete graph, and the meaning of the graph lines presented in relation to the possible domains of the functions. Questions 2 and 3 aim to lead students to generalize a procedure to figure out the intersection point of two functions and to generalize the relation between the solutions of an equation and the graphical representation of those two functions (principle (ii)). Question 2 allows the students to use different solving strategies, like trial and error or the sought procedure (principle (iv)). Question 2 also asks for a justification and an implicit justification is asked in question 3, as students are not expected to simply indicate the day in which the plants have the same height (principle (iii)). The design principles for the teacher actions, regarding both students' work and whole class discussions, are considered in the lesson plan and meant to be present throughout the lesson.

Mary received a plant as a gift and she kept a record of its growth. John thought it was a really nice idea and, a couple of days later, bought a plant and also kept a record of its growth. The functions that follow represent the height of both plants in their first days with the students:

$$
\text { Mary's plant: } m(x)=0.4 x
$$$$
\text { John's plant: } j(x)=0.2 x+2.2
$$

Observe the graph of these functions:

1. In which day do the plants have the same height?

2. Consider the comment: "Graphs are not necessary for us to know in which day the plants have the same height. Knowing the functions that represent the growth of each plant is enough to verify when they are equal". What

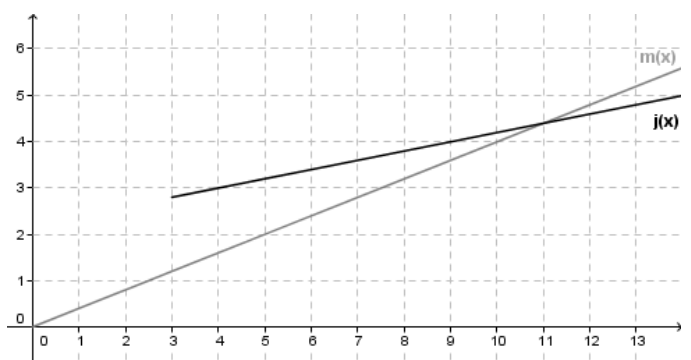
would be another way to figure the day in which the plants have the same height? Justify your answer.

3. Catherine, Richard, and Ingrid did an identical register of the growth of their plants, which can be represented by the following functions: 


Catherine's plant: $c(x)=0.6 x+2 \quad$ Richard's plant: $r(x)=2+\frac{3}{5} x$
Ingrid's plant: $i(x)=1+0.6 x$
3.1. In which day Catherine's plant has the same height as Richard's plant? And as Ingrid's plant?
3.2. Consider your previous results. Which relationship can you establish between the graphs of functions
$c$ and $r$ ? And of function $c$ and $i$ ?

Fig. 2 Proposed task.

Assuming generalizing and justifying as central processes of mathematical reasoning, this task aimed that students would (1) produce a generalization of an algebraic procedure to determine the $x$ intercept of any two functions, by equating the algebraic expressions of both functions and solving that equation, and (2) justify their responses and strategies. As students already know how to figure out the intersection point of two functions given in graphic representation, they are expected to achieve these two aims based on their knowledge about functions and equations, and based on the relations between these two mathematical concepts. This task led to several moments of whole class discussion, from which we present five episodes. The first episode concerns an initial discussion about questions 1 and 2, whereas the second addresses the strategy used in question 2 . The third and fourth episodes concern the use of the procedure generalized in question 2 and interpreting the results with the lenses of question 3.2. The last episode addresses a justification that goes beyond the aim of the task.

\subsection{Formulating a strategy}

The episode. The teacher begins the lesson by introducing the task to the students and asks them to work on it. Some students immediately answer question 1, which leads to a first whole class discussion regarding the functions involved and their representations. Then, the students tackle the task in pairs. As they work, the teacher perceives that many have difficulty in dealing with question 2 . She calls the students' attention and invites the class to engage in a whole class discussion, rephrasing the question without reducing its level of challenge (principle (a)):

If you had never seen the graphs and if you only had both algebraic expressions, this two [points both expressions], how would you... You have seen the graph and realized straight away that it was on the $11^{\text {th }}$ day, why?

The teacher suggests an interpretation to the statement of question 2, but she soon realizes that it would be better to begin with question 1 as the students had ease in solving it. She encourages sharing ideas (principle (d)), challenging students to justify an answer (principle (b)) that she knows the whole class achieved.

Iris tries to address this challenge:

Iris: Through the graph.

Teacher: Why through the graph?

Ruben: Because they get together.

Abel: Because it's there [pointing the point of intersection]! 
Iris refers to the graph as a means to obtain the answer, but her justification is incomplete as she does not refer to the point of intersection. The teacher values her partial contribution by using her answer (principle (e)) and, asking why, she also challenges once more the students to go further on justifying this answer (principle (b)).

As the students refer to the point where both functions intersect, thus presenting the sought justification, the teacher suggests a more accurate form of saying it (principle (f)), thus validating this justification:

They intersect, isn't it? They touch each other here [pointing the point of intersection], that corresponds to number $11, \mathrm{OK}$.

Given the agreement on the response to question 1 and the validation of the explanation of "why", the teacher proceeds to the discussion of question 2. She challenges the students to come up with a strategy to solve the question (principle (d)). However, this challenge is not successful, as the students are still confused and keep silent, so she begins to guide them to formulate a strategy asking what the aim of the question is. Still, this guiding action is not enough to lead the students in formulating a strategy and the teacher goes further on guiding. Even so, this is still not enough as the students provide no answer.

Although these actions do not yield the sought students' participation, they open the path for them to present their ideas. Abel intervenes:

Abel: I have $0.4 x$ less 0.2 .

Teacher: $0.4 x \ldots$ Oh, but you are already solving what?

Abel: The expressions.

Teacher: Say it.

Abel: $0.4 x$ equals $0.2 x$ plus 2.2 .

At the beginning of his contribution, Abel does not answer the actual challenge posed by the teacher, but directly tackles question 2. The teacher encourages him in sharing his idea (principle (d)), supporting him to complete his contribution (principle (e)). This teacher action leads Abel to present the idea that one should equal one function to the other $(m(x)=j(x))$, identifying the aimed strategy to solve question 2 .

This strategy entails the generalization of the sought procedure in this task. Thus, the procedure of equating the algebraic expressions of both functions is settled by Abel with respect to the functions $m(x)$ and $j(x)$ which allows the students to later use this procedure in any other set of two functions. However, as the procedure is not explicitly stated, the teacher challenges Abel by asking for a justification of his statement (principle (b)):

Teacher: Why do we solve this? Why do I find what I want if I solve this?

Abel: Because the result is 11 . 
Abel presents an invalid justification. By posing another question, the teacher informs that his statement is not enough as a justification (principle (c)) and guides the students towards a proper justification (principle (b)):

Teacher: But why do we make [those algebraic expressions] equal?

Mariana: To verify if it is equal.

Teacher: To verify if they are equal?

Abel: No, if they intersect.

Mariana provides an invalid justification, but, this time, by rephrasing her statement, the teacher challenges the students to validate her answer (principle (c)). At his point, Abel identifies that Mariana's statement is not valid, presenting a more accurate, although incomplete, justification about why one has to establish an equality between the two algebraic expressions.

Further analysis. In this discussion episode, the teacher perceives the students' difficulties in dealing with question 2 . This leads her to rephrase the question in order to engage the students in it without reducing the level of challenge (principle (a)). Then, throughout the discussion, the teacher, either by challenging or supporting actions, leads the students to clarify and deconstruct incorrect or incomplete contributions (principle (e)) and provides encouragement to share ideas (principle (d)). Principle (b) is rather salient, as the teacher questions the students several times in order to lead them to explain "why". To tackle this principle, the teacher mostly relies on challenging actions. Nevertheless, when the students' justifications are incorrect or incomplete, she also tackles this principle with guiding actions. Regarding validating students' justifications (principle (c)), the teacher either validates or invalidates students' justifications by relying on informing actions, or asks students to do so by challenging them. In this last case, she also highlights the need for justification (principle (f)), suggesting it in a more accurate mathematical language. In this episode, the generalization of the sought procedure emerges due to Abel's strategy. This generalization, despite being unstated by either the students or the teacher, becomes more prominent as the teacher asks for a justification of Abel's strategy. Thus, in this episode, the students have several opportunities to justify that are prompted by the features of the task and by the teacher's actions based on the design principles, as well as by the students' responses to those actions.

\subsection{Focusing on the procedure and triggering an unexpected generalization}

The episode. The discussion goes on, aiming to highlight that the point where the two lines intersect is also the point in which the two expressions have the same value. When this discussion episode comes to an end, Tito intervenes, conjecturing, without solving the equation, that solving it would yield the same result as looking at the graph and finding the point where the lines intersect:

Teacher: Oh, it is the day, is the day in which Mary's plant height equals John's plant height. Isn't it? So, solve it to check if we looked properly to the graph.

Tito: But teacher... It will be 11 . 
At this point the teacher challenges the students to solve the equation all together, encouraging the sharing of ideas (principle (d)). She alternates between guiding and supporting actions, and with the participation of several students, the discussion entails the solution of the equation $(x=11)$.

Even though the strategy is established and verified by solving the equation, the teacher allows Tito to share his solving process (principle (d)):

Tito: I have done it by multiplying by 5 [referring to a step where Abel divided by 0.2 in $0.2 x=$ 2.2].

Abel: Which yields the same thing!

Tito: 0.2 times 5 is $1 \ldots$ That also leads to $x$. And then 2.2 times 5 also leads to 11 .

Teacher: You multiplied both members by 5. Is this what you are saying?

Tito: Yes.

The teacher is not expecting Tito's solving strategy, so she supports the student's answer, asking for his confirmation (principle (e)). At this point, Abel quickly replies to Tito's statement. He does not refer to the particular result of 0.2 multiplied by 5 and of 0.2 divided by 0.2 , Abel refers to a general situation, relying on the generalization that dividing by 0.2 or multiplying by 5 yields the same result for any given number.

In order to focus the class on this new strategy of solving the equation, the teacher informs the students about Tito's idea, and then she decides to go further (principle (g)), challenging the students to explain why this idea is pertinent (principle (b)), aiming on focusing on multiplication by 5 as equivalent to division by 0.2 , as some students might not be aware of this generalization regarding the relationship between the two operations:

Teacher: Very well. Pay attention to what Tito is saying, that is a quite interesting alternative to division by 0.2 . Tito is saying that when he gets to this step [0.2x $=2.2]$ instead of dividing by $0.2 \ldots$ Why did Tito make this decision?

Tito: I don't know, as I've seen a decimal, I thought that multiplying would be easier.

As the teacher realizes that the students do not have the tools to respond to her challenge, she reinforces Tito's idea, suggesting a clarification of the procedure that he followed (principle (e)). Also, she validates and highlights his procedure and then concludes synthesizing the strategy to answer question 2, using suggesting actions.

Further analysis. In this episode, the teacher seeks to further develop the discussion regarding the strategy to solve question 2, which is a central aim of this task. To do so, she mainly uses guiding actions while encouraging the students' participation (principle (d)). Despite the fact that the aim of question 2 was already achieved, the teacher supports the discussion of a different way of solving the equation proposed by Tito. By allowing the students to present their ideas and particularly by considering their partial contributions (principle (e)), an unexpected solving process and a consequent generalization emerge from the discussion. While tackling this unformulated generalization, the teacher also tries to 
engage the students in justifying it by using challenging actions that aim to obtain the explanation of why (principle (b)) and also to go beyond the task (principle (g)). However, she soon realizes that the students do not have the tools to respond to this challenge, withdraws it, and just summarizes the solution proposed. This episode also allows the students to focus on the procedure to be generalized in this task.

\subsection{Using the generalization and interpreting results}

The episode. After discussing questions 1 and 2, the students work autonomously on question 3. During this work, the teacher monitors the students mostly asking them to explain what they are doing, without reducing the level of challenge of the question (principle (a)). With the tools developed in the previous discussion, most of the students have ease on using the generalization that emerged on the discussion of question 2, writing equations by equating the expressions given in the statement of question 3 . After the students' autonomous work, the teacher asks one of them to write the equations on the blackboard and invites the students to share their ideas about question 3 (principle (d)). Abel intervenes:

Abel: Every day [the height of plants] is going to come across each other.

Teacher: Explain it better, you're speaking in coded language.

Francisco: They are going to intersect each other.

The teacher reacts to Abel's contribution with a challenging action, aiming to go further on his use of the mathematical language (principle (e)). However, it is another student, Francisco, who responds to the challenge.

In this discussion, the teacher begins by letting the students share their ideas (principle (d)), but then, she feels the need to refocus the discussion on the procedure used to solve question 3.1. As the students, in their autonomous work, easily used in question 3.1 the generalization obtained in question 2, the teacher opts to inform the students about this procedure (principle (f)) and to guide them in validating the numerical equality obtained (principle (c)). By doing so, the teacher uses the limited time of the discussion to focus on making conclusions about the result of applying the procedure instead of focusing on the procedure itself. Thus, the teacher guides the students to draw a conclusion from that numerical equality, aiming to supplement their previous answers (principle (e)):

Teacher: And that leads us to conclude what?

Abel: That they are going to come across each other every day.

Abel's contribution provides the answer that the teacher wanted. However, the teacher informs the students validating this conclusion (principle (c)) and challenges them to go beyond the question (principle $(\mathrm{g})$ ), by asking the number of solutions of the equation:

Teacher: They intersect every single day, OK? How many solutions has this equation $[0.6 x+2=$ $\left.2+\frac{3}{5} x\right] ?$

Several students: Infinite. 
Teacher: Infinite. That means that this plants, Richard's [plant] and Catherine's [plant] always were growing...

Abel: At the same height.

Teacher: Since the beginning that they have the same height.

The students easily respond to this challenge and the teacher guides them to establish a relation between the number of solutions and its meaning in this context, and she concludes by informing the students of the validity of this relationship (principle (c)).

At this moment, the teacher decides to skip the second part of question 3.1 and challenges the students to consider the first part of question 3.2, about the plants of Catherine and Richard, to establish a more significant relationship between those two aspects of the same situation:

Teacher: Graphically, this is... This $[c(x)]$ is a line and this $[r(x)]$ another line and graphically, how will those lines be?

Iris: Equal.

Teacher: Equal, what do you mean?

Iris: [Gesturing] In the same place.

Tito: Overlapping.

The students had already indicated that these two plants had the same height every day. Therefore, they easily realize how the graphs will be, needing only some guidance from the teacher in order to better communicate it (principle (e)). In doing so, students obtain a particular example that provides them the opportunity to later generalize that two functions that have an infinite number of intersection points are represented by coincident lines (which students, with some teacher guidance, generalize in the next lesson).

The teacher ends this discussion episode with a summary, informing the students about this particular example (principle (f)), representing what they discussed in GeoGebra and giving some extra information by suggesting that the graphs should be line segments and not straight lines.

Further analysis. The discussion about question 3 is structured mainly by guiding and challenging actions that consider or promote partial contributions from the students (principle (e)) followed by guiding or informing actions that highlight the validity of those contributions (principle (c)). This path of actions begins with an inviting action that encourages the students to participate in the discussion (principle (d)). Despite this central path, the teacher begins by highlighting the generalization of the sought procedure (principle (f)) relying on guiding and (mainly) on informing actions. Also, by challenging the students to go beyond question 3.1 and by connecting it to question 3.2 (principle (g)), she provides the students with the groundwork to later generalize that, if the procedure used in this task yields an infinite number of solutions, then the graphical representations of those functions coincide.

\subsection{Using the procedure once more and interpreting further results}

The episode. The discussion comes back to the second part of question 3.1 that aims to compare the height of the plants of Catherine and Ingrid. The beginning of this discussion is similar to that of episode 
4.3, using mostly informing and guiding actions. When a student finishes solving the equation on the blackboard, the teacher informs the whole class by highlighting the result of this equation $(2=1)$. However, Abel reacts stating that he achieved a different result $(0 x=-1)$. The teacher allows Abel to share his solving process (principle (d)) albeit informing that it leads to the same conclusion. She chooses to guide the students towards Abel's solving process, once again alternating between guiding and informing actions (principle (a)), leading the students to reach the expression $0=-1$. Still, Abel is not satisfied with the arguments presented by his colleagues and by the teacher and claims that the two solutions are different:

Abel: But it is $0 x$ straight away, teacher.

Teacher: Sure, because you did $0.6 x$ minus $0.6 x, 0 x$. Done. And you took 2 from the first member. Abel: But my thing $[0 x=-1]$ is possible, this $[0=-1]$ isn't!

Faced with Abel's contribution, who keeps stating that this outcome is not the same as the former, the teacher challenges him to justify the validity of his statement (principle (b)) in order to address his misconception (principle (e)):

Teacher: Is yours possible?

Francisco: No it isn't.

Teacher: $0 x$ equals -1 ?

Several students: No it is not [equal].

Abel does not respond to the teacher's challenge, but Francisco does, presenting an incomplete justification. Faced with a guiding question, several other students also respond with the same incomplete justification. Therefore, the teacher decides to guide the students in further justifying their answer (principle (b)):

Teacher: So, tell me, what's the value of $x$ that transforms $0 x$ in -1 ?

Francisco: Because zero times anything is always zero.

At this moment of the discussion Francisco properly justifies that Abel's equation is impossible to solve relying on known properties of numbers. Then the teacher focuses the students' attention on that justification by informing them (principles (c) and (f)) and she challenges Abel to re-evaluate his statement, which the student accurately does.

Similarly to the discussion of the previous situation of comparing the height of the plants of Catherine and Richard, the teacher informs that the equation is impossible and then she challenges the students to go beyond the question by identifying how many solutions this equation has (principle $(\mathrm{g})$ ). Also, following the same path as before, the teacher challenges the students to answer to the second part of question 3.2, informing the class about the ideas that emerge. Unlike what happened earlier, the students struggle to figure that the graphs of both functions are parallel, proposing, for example, non- 
parallel line segments that do not intersect. The discussion goes on until Iris presents another possibility for a graphical representation of both functions:

Iris: Teacher, they could be both like this [gestures two parallel straight lines]...

Teacher: They are going to be...

Vasco: Parallel.

As the teacher begins to inform the students about what Iris is trying to say, Vasco corroborates the idea of her colleague using a proper mathematical term. As in the previous discussion episode, the students gain some insights that may lead them to generalize that two functions with no points of intersection have parallel representations. At this moment of the lesson, the whole task had been discussed.

Further analysis. In the beginning of this episode, the teacher uses informing and guiding actions to engage the students (principle (d)) in solving the equation that might lead to the intersection of the two functions tackled. In doing so, by relying on guiding actions and using informing actions mostly to rephrase students' answers, she does not reduce the level of challenge of the task (principle (a)). Due to an unexpected statement from Abel, the teacher challenges and guides the students by asking for a justification and further alternative justifications (principles (b) and (e)), leading them towards an appropriate justification of Abel's invalid statement. In order to synthesize this justification (principle (f)), the teacher highlights its validity (principle (c)), with informing actions. When comparing the height of the plants of Catherine and Richard, she challenges the students to go beyond the procedure (principle (g)) by identifying the number of solutions of the equation, providing the students with the ideas that may lead them to generalize that if the equation does not yield any solution, then the tackled functions have parallel graphic representations.

\subsection{Eliciting a further justification}

The episode. Despite the fact that the aims of the task are already achieved, the teacher decides to challenge students to go further on their answers (principle $(\mathrm{g})$ ) by asking them to justify the parallelism between the lines representing these functions:

Teacher: Why do they have to be parallel?

Vasco: Because the plants of Catherine and Richard begin with 2 centimeters and then always grow [in the same way], and Ingrid's plant begins with 1 centimeter and grows in the same way.

Vasco refers to the growth of plants, justifying the parallelism with the slope of the functions. As this justification is not completely explicit, the teacher chooses to guide the students to present an alternative conclusion (principle (b)):

Teacher: And is there any other option for the straight lines not to intersect but to be parallel? Several students: No. 
Teacher: No, then, in a graph, we have this [presents the GeoGebra representation]. And I also want Vasco to share something really interesting that he noticed.

As the students easily answer to this guiding action from the teacher, she informs them that their conclusions are valid (principle (c)). Only then she challenges Vasco to explain his previous justification (principle (b)). He responds to the challenges in the following way:

Vasco: [Reading his answer] As Catherine's and Richard's [lines] begin with the same height and as both plants grow at the same rate, they are not going to intersect. Ingrid's, as it grows every day...

Teacher: They are not going to intersect?!

Vasco: Obviously, they are already overlapped.

The teacher had monitored Vasco's autonomous work, so, when the student states that the functions do not intersect, she reacts by challenging him to justify that statement (principle (b)). However, it was just a matter of what Vasco meant with "intersect", as he seems to be considering that this only occurs if the graphs of two functions have a few number of points in common. Complementing his previous contribution, Vasco justifies that as two functions have the same slope they have coincident representations, this time explicitly referring to the slope as he states "the same rate".

When Vasco justifies his statement regarding that the plants' height is not going to intercept, the teacher informs the class about that justification, validates it (principle (c)), and guides him to proceed:

Teacher: Oh, OK, they overlap. And the other two?

Vasco: Ingrid' plant, as it grows every day the same height than the other two and begins with less a centimeter... Is going to be always smaller.

Teacher: It will always be smaller, and that is why they never intersect. Exactly.

The procedure that Vasco used for the first two functions is adapted to the new conditions provided by the second two functions, which entails a generalization of the procedure that allows the student to relate the slope of the functions with their relative position. This discussion ends with another informing action from the teacher that summarizes the relation between the height of the plants of Catherine and Ingrid and their graphs. As the time was over, there was no opportunity in this lesson to discuss possible generalizations regarding the relationship between the relative position of the graphs of two functions, and, either the number of solutions of an equation that equals those functions, or the slope of those functions.

Further analysis. When the discussion of the task comes to an end, the teacher challenges the students by asking them to justify the parallelism between the graphs of those particular functions (principle (g)). Then, relying on guiding and informing actions, she asks for justifications (principle (b)) and indicates if students' justifications are valid or not (principle (c)) using informing actions. Also, the generalization in this episode differs from the ones envisioned in previous episodes as it relates to a 
procedure that consists of using the notion of slope in order to figure out the relative position of the lines that represent the functions, going beyond the scope of the task.

\section{Discussion}

The whole class discussion episodes that we presented illustrate the relations between design principles and students' reasoning processes. These episodes provide a variety of insights regarding teacher actions to stimulate generalizations and justifications. Regarding generalization, they highlight paths towards sought generalizations (episode 4.1), towards using or providing unexpected generalizations (episodes 4.2 and 4.5 , respectively), or towards providing students with examples that might later be generalized (episodes 4.3 and 4.4). About justification, the episodes illustrate paths that provide the students with opportunities to justify and complete their justifications (episodes 4.1 and 4.4), to move from invalid to incomplete justifications (episode 4.1), to provide alternative justifications (episode 4.5), or paths where there is the need to withdraw the challenge as students do not have the tools to justify (episode 4.2).

Moreover, altogether, these whole class discussion episodes provide a general understanding regarding the design principles established and their relation to students' mathematical reasoning processes. Regarding the design principles for tasks, we note that the exploratory questions provided fertile ground for the students to use particular examples to make generalizations such as figuring a procedure to find where two functions intersect. This use of particular examples, as in previous studies (Kieran 2007), proved to be a fruitful setting for mathematical learning. The proposed task, designed in collaboration with the teacher, focused on promoting students' learning about linear equations by making sense of mathematical properties. Despite that most of the students' generalizations and justifications emerged from the discussion and not directly from the task, all the situations in the whole class discussion that we analyzed were prompted by the task.

Regarding the design principles for teacher actions, all of them appear to be relevant to enhance students' mathematical reasoning. Moreover, particular design principles combined with more general teacher actions seem to lead to students' generalizations and justifications. As highlighted in previous studies (Franke et al. 1999; Kosko et al. 2014), a single action is not enough to promote situations that elicit mathematical reasoning processes. There is the need to focus on sequencing of actions. The framework to analyze teacher actions that distinguish between inviting, informing/suggesting, guiding/supporting, and challenging actions (Ponte and Quaresma 2016) is useful to understand the paths of actions, and supported the reframing of the design principles. A principle that is most noticeable in the discussion is asking to explain the "why" or to present an alternative justification, but by itself may not lead to a justification. However, as illustrated in this paper, if this "why" question (which is usually a challenging action) is followed by guiding actions, some justifications are likely to emerge, either valid or invalid. Often, a teacher's action within this principle is followed by an action related to validating a statement, where the teacher tends to do informing actions and rarely uses challenging actions. Challenging actions also seem to have the potential to engage the students in justifying. Within similar situations or even in the same ones, the principle related to highlighting generalizations and justifications was enacted. Regarding generalization, beginning by inviting students and also by challenging or guiding them to participate, focusing on encouraging the students to share their ideas, may elicit unexpected generalizations. Also, challenging students to go beyond the task, usually through a central challenging 
action, followed by guiding and informing actions, leads the students to generalize. Thus, in designing an intervention that aims to develop students' mathematical reasoning, a combination of design principles seems to be useful, as isolated principles might not be enough to achieve such aim.

\section{Conclusion}

This study provides insights into how reasoning processes may emerge in whole class discussions carried out in a challenging learning environment. First, there is a major relevance of the structure of the intervention itself. Here, the teacher strong engagement is essential to overcome what Kosko et al. (2014) identified as a stark contrast between what researchers and teachers value as the actions more effective in promoting students' mathematical reasoning. Second, the whole class discussion itself presented the students with opportunities to enhance their reasoning processes, a central aspect of an environment that allows them to make sense of mathematics (Brodie, 2010). And third, within this environment, these reasoning processes tend to emerge if some action paths are followed by the teacher. When aiming to enhance students' mathematical reasoning, a central challenging action may elicit a generalization or a justification, but most often it requires several follow-up actions from the teacher.

A further study that will be interesting to undertake may address what new steps may be taken to make proofs come to life in the mathematics classroom. Moving from generalizations and justifications to proofs is not immediate either to the students or the teacher. In fact, a single intervention such as this is not enough to provide the students all the tools necessary to develop and understand proof (Jahnke and Wambach, 2013). Also, as highlighted by Stylianides (2007), teachers need guidance and resources in order to be able to cultivate proof and proving among their students. As a first step, as referred by Brodie (2010), it is important to share interventions regarding mathematical reasoning and what those interventions may achieve. In the current intervention, the set of presented principles constitutes a promising environment to develop students' ability to make appropriate generalizations and justifications. And, by generalizing and justifying, students develop their mathematical reasoning and can be better equipped to later deal with mathematical proof.

\section{Acknowledgments}

This work is supported by national funds through FCT - Fundação para a Ciência e Tecnologia by a grant to Joana Mata-Pereira (SFRH/BD/94928/2013).

\section{References}

Becker, J.R., \& Rivera, F. (2005). Generalization strategies of beginning high school algebra students. In H.L. Chick \& J.L. Vincent (Eds.) Proceedings of the $29^{\text {th }}$ PME Conference (vol. 4, pp. 121-128). Melbourne: PME.

Ball, D., \& Bass, H. (2003). Making mathematics reasonable in school. In J. Kilpatrick, W. Martin, and D. Schifter (Eds.), A research companion to principles and standards for school mathematics (pp. 27-44). Reston, VA: NCTM.

Boaler, J. (2010). The road to reasoning. In K. Brodie, Teaching mathematical reasoning in secondary school classrooms (pp. v-vii). New York, NY: Springer.

Brodie, K. (2010). Teaching mathematical reasoning in secondary school classrooms. New York, NY: Springer.

Brousseau, G., \& Gibel, P. (2005). Didactical handling of students' reasoning processes in problem solving situations. Educational Studies in Mathematics, doi:10.1007/s10649-005-2532-y 
Carraher, D., Martinez, M., \& Schliemann, A. (2008). Early algebra and mathematical generalization. ZDM, doi: 10.1007/s11858-007-0067-7

Christiansen, B., \& Walther, G. (1986). Task and activity. In B. Christiansen, A. G. Howson, \& M. Otte (Eds.), Perspectives on mathematics education (pp. 243-307). Dordrecht: D. Reidel.

Cobb, P., Confrey, J., diSessa, A., Lehrer, R., \& Schauble, L. (2003). Design experiments in educational research. Educational Researcher, doi:10.3102/0013189X032001009.

Dörfler, W. (1991). Forms and means of generalization in mathematics. In A. Bishop, S. Mellin-Olsen \& J. van Dormolen (Eds.), Mathematical knowledge: Its growth through teaching (pp. 63-85). Dordrecht: Kluwer.

Francisco, J.M., \& Maher, C.A. (2011). Teachers attending to students' mathematical reasoning: lessons from an after-school research program. Journal of Mathematics Teacher Education, doi:10.1007/s10857-010-9144-x.

Franke, M.L., Webb, N., Chan, A., Ing, M., Freund, D., \& Battey, D. (2009). Teacher questioning to elicit students' mathematical thinking in elementary school classrooms. Journal of Teacher Education, doi:10.1177/0022487109339906

Jahnke, H.N., \& Wambach, R. (2013). Understanding what a proof is: a classroom-based approach. ZDM Mathematics Education, doi:10.1007/s11858-013-0502-x

Kieran, C. (2007). Learning and teaching algebra at the middle school through college levels: Building meaning for symbols and their manipulation. In F. Lester (Ed.), Second handbook of research on mathematics teaching and learning (pp. 707-762). Reston, VA: NCTM.

Kosko, K., Rougee, A., \& Herbst, P. (2014). What actions do teachers envision when asked to facilitate mathematical argumentation in the classroom? Mathematics Education Research Journal, doi:10.1007/s13394-013-0116-1

Krussel, L., Edwards, B., \& Springer, G.T. (2004). The teacher's discourse moves: A framework for analyzing discourse in mathematics classrooms. School Science and Mathematics, doi:10.1111/j.1949-8594.2004.tb18249.x

Lannin, J., Ellis A.B., \& Elliot, R. (2011). Developing essential understanding of mathematics reasoning for teaching mathematics in prekindergarten-grade 8. Reston, VA: NCTM.

Pólya, G. (1954). Mathematics and plausible reasoning: Induction and analogy in mathematics (Vol. I). Princeton, NJ: Princeton University Press.

Ponte, J.P. (2005). Gestão curricular em Matemática. In GTI (Ed.), O professor e o desenvolvimento curricular (pp. 11-34). Lisboa: APM.

Ponte, J.P., \& Quaresma, M. (2016). Teachers' professional practice conducting mathematical discussions. Educational Studies in Mathematics, doi:10.1007/s10649-016-9681-z

Reid, D. (2002). Conjectures and refutations in grade 5 mathematics. Journal for Research in Mathematics Education, 33(1), 5-29.

Rivera, F., \& Becker, J. (2009). Algebraic reasoning through patterns. Mathematics Teacher in the Middle School, 15(4), 213-221.

Ruthven, K. (1989). An exploratory approach to advanced mathematics. Educational Studies in Mathematics, doi:10.1007/BF00315610

Sherin, M. (2002). A balancing act: Developing a discourse community in a mathematics classroom. Journal of Mathematics Teacher Education, doi:10.1023/A:1020134209073

Stein, M.K., Engle, R., Smith, M., \& Hughes, E. (2008). Orchestrating productive mathematical discussions: Five practices for helping teachers move beyond show and tell. Mathematical Thinking and Learning, doi:10.1080/10986060802229675

Stylianides, A. (2007). Proof and proving in school mathematics. Journal for Research in Mathematics Education, 38(3), 289-321.

Sowder, L., \& Harel, G. (1998). Types of students' justifications. Mathematics Teacher, 91(8), 670-675. 
Wood, T. (1999). Creating a context for argument in mathematics class. Journal for Research in Mathematics Education, 30(2), 171-191. 